\title{
Voltammetric sensor for electrochemical determination of the floral origin of honey based on a zinc oxide nanoparticle modified carbon paste electrode
}

\author{
Kamalika Tiwari $^{1}$, Bipan Tudu ${ }^{1}$, Rajib Bandyopadhyay ${ }^{1,2}$, Anutosh Chatterjee ${ }^{1}$, and \\ Panchanan Pramanik ${ }^{3}$ \\ ${ }^{1}$ Department of Instrumentation and Electronics Engineering, Jadavpur University, Kolkata, India \\ ${ }^{2}$ Laboratory of Artificial Sensory Systems, ITMO University, Saint Petersburg, Russia \\ ${ }^{3}$ Institutes of Applied Science and Humanities, GLA University, Mathura, India \\ Correspondence: Kamalika Tiwari (tiwari.kamalika@gmail.com)
}

Received: 6 October 2017 - Revised: 27 March 2018 - Accepted: 27 March 2018 - Published: 26 April 2018

\begin{abstract}
A new methodology based on cyclic voltammetry using a chemically modified electrode has been developed for the discrimination of the floral origin of honey. This method involves an electronic tongue with an electrochemical sensor made from a carbon paste (CPs) electrode where zinc oxide $(\mathrm{ZnO})$ nanoparticles are used as an electroactive binder material. The bare CPs electrode is evaluated for comparison. The electrochemical response of the modified electrode in 50 samples of five different floral types of honey has been analysed by the cyclic voltammetric technique. The voltammograms of each floral variety of honey reflect the redox properties of the $\mathrm{ZnO}$ nanoparticles present inside the carbon paste matrix and are strongly influenced by the nectar source of honey. Thus, each type of honey provides a characteristic signal which is evaluated by using principal component analysis (PCA) and an artificial neural network (ANN). The result of a PCA score plot of the transient responses obtained from the modified carbon paste electrode clearly shows discrimination among the different floral types of honey. The ANN model for floral classification of honey shows more than $90 \%$ accuracy. These results indicate that the $\mathrm{ZnO}$ nanoparticles modified carbon paste ( $\mathrm{ZnO}$ Nps modified $\mathrm{CPs}$ ) electrode can be a useful electrode for discrimination of honey samples from different floral origins.
\end{abstract}

\section{Introduction}

During the last few years, revealing the floral origin of honey has been a subject of many studies because of its potential in determining nutritional and medicinal aspects (Mandal and Mandal, 2011; Bueno-Costa et al., 2015; Chua et al., 2012). It is reported that honey possesses a high therapeutic value, particularly in treating ailments like heart and inflammatory diseases (Oryan et al., 2016; Yousuf et al., 2016). It is a naturally sweet food produced by honeybees from the nectar of plants and is mainly composed of sugar, enzymes, organic and phenolic acids, vitamins, minerals, trace elements and pollens (Missio da Silva et al., 2016; Kadri et al., 2017). Determination of pollen helps in identifying the nectar source of honey. Depending upon the source, the composition of honey varies (Ball et al., 2007). The physiochemical attributes of honey, i.e. colour, flavour, and taste vary according to nectar source and climate (El Sohaimy et al., 2015; Karabagias et al., 2014a, b). In recent years, with an increase in consumer demand for authentic honey, more and more adulterated honey has entered the market. Further, the EU and Codex standard for honey strictly states that the floral and geographical origins must be declared before commercialization to assure honey quality and fraud labelling (Codex Alimentarius Commission, 2001). Thus, a particular monofloral honey is associated with commercial value and floral origins of honey need to be assessed (Ozcan and Olmez, 2014).

Traditionally, botanical identification of honey is carried out by microscopic inspection (Anklam, 1998; Feas et al., 2010). However, the method involves limitations in terms of sample preparation, time consumption and the need for expertise in pollen identification. This has led to a search 
for alternate methods of honey analysis using instrumental techniques (Siddiqui et al., 2017) such as atomic absorption spectroscopy (AAS) (Pohl et al., 2011), capillary electrophoresis (Jiang et al., 2015), high-pressure liquid chromatography (HPLC), thin layer chromatography (TLC) (Cavazza et al., 2013; Ares et al., 2015; Rezic et al., 2015), gas chromatography and mass spectrometry (GCMS) (Soto et al., 2015), isotope ratio mass spectrometry (IRMS) (Dinca et al., 2015), electro-spray mass spectrometry (ESMS) (Kaskoniene et al., 2015), nuclear magnetic resonance (NMR) spectroscopy (Zheng et al., 2015), near-infrared (NIR) spectroscopy (Woodcock et al., 2007) and many more. These are separation methods, i.e. constituents are first separated and then chemical fingerprinting is carried out for different floral markers. They involve costly equipment, complex sample preparation and are time-consuming. It is difficult to analyse and correlate many samples together in a short span of time.

Recently, newer methods for the identification of the floral origin of honey using the electronic tongue have been formulated (Wei and Wang, 2011, 2014; Dias et al., 2015; Sousa et al., 2014; Ulloa et al., 2013; Belay et al., 2017). The electrochemical sensors of the electronic tongue are the most effective alternative for qualitative assessment as honey constitutes a combination of various electroactive compounds. These electrochemical methods have achieved more significance due to low cost, rapid performance, high selectivity and sensitivity. A multisensory system based on nonselective electrochemical sensors forms an electronic tongue (Peris and Gilabert, 2016; Legin et al., 2003). The sensors play a pivotal role in the performance of an electronic tongue. In the electrochemical analysis, the voltammetric technique has been regarded as the most popular technique in the complex liquid analysis because of high sensitivity, simplicity and good signal to noise ratio (Ceto et al., 2017; Wei and Wang, 2013; Apetrei and Apetrei, 2016). Voltammetric sensors as working electrodes provide information about the redox processes occurring in the solution when a potential is applied. Such electrodes provide voltammograms, which show the redox processes corresponding to the electro-active material chosen. The active carbon paste is one of the most interesting sensor materials for fabrication of chemical sensors because of ease in obtaining the material at low cost and its amenability to modification depending upon the target analyte. Carbon paste electrodes have received worldwide attention due to their unique chemical stability, high aspect ratio, and high specific surface area and the fact that they can be used at both positive and negative potentials depending upon the support electrolyte (Svancara and Schachl, 1999; Svancara et al., 2001, 2008; Rodriguez-Reinoso et al., 1998). But, bare CPs electrodes are prone to the problem of electrode fouling and slow electron transfer reaction. Depending upon different applications, bare electrodes are modified with selective compounds. It is for these reasons that chemical modification of the electrode with desired characteristics is in high demand. The chemically modified electrodes are widely used as voltammetric sensors due to versatility and unique electrochemical and electro-catalytic properties. Past studies report that various types of modifiers (Skrzypczynska et al., 2016; Terbouche et al., 2016) are available among which nanocrystalline materials are more advantageous because of their large surface area and high thermal and chemical stability. The additions of nanomaterials with common CPs electrodes are useful for catalysis and are advantageous in making sensors. Conversely, $\mathrm{ZnO}$ nanoparticles have advantages such as narrow size distribution, efficient surface modification, and desirable biocompatibility (Bijad et al., 2013). Nanomaterial modified electrodes have the high electron transfer ability (Fernandez-Garcia and Rodriguez, 2007). In particular, metal oxide nanomaterials exhibit unique physical and chemical properties due to their size and high density at the corner or edge surface sites. Metal oxides are used for both their redox and acid/base properties in the context of absorption and catalysis (KarimNezhad et al., 2016; Afghani et al., 2016; Tavakolian et al., 2016).

In the present work we report a simple, low-cost and sensitive voltammetric electrode based on a $\mathrm{ZnO}$ nanoparticle modified CPs electrode and the unmodified CPs electrode for comparison, fabricated for the determination of the floral type of honey sample. The electrochemical behaviour is investigated using the cyclic voltammetry technique. The advantages of incorporating nanoparticles into the CPs electrode are investigated for the discrimination of the floral type of honey. The sensor exhibits acceptable reproducibility and stability for determination of the floral source of honey. The response of the electronic tongue is treated with principal component analysis (PCA) to derive the clustering capability for each of the electrodes and ANN has been used for determining the classification of honey samples.

\section{Materials and methods}

\subsection{Apparatus and reagents}

Cyclic voltammetric (CV) experiments are performed using an electronic tongue set-up. The set-up comprises a working electrode, an $\mathrm{Ag} / \mathrm{AgCl}$ reference electrode (saturated KCL, Gamry Instruments Inc.) and a platinum wire as the auxiliary electrode (PH Ionics, India). The working electrode is the unmodified CPs electrode or modified CPs electrode. A pH meter (TANOTIS PH-2011) is used for $\mathrm{pH}$ measurement. All the experiments are conducted at room temperature $\left(25 \pm 1^{\circ} \mathrm{C}\right)$.

Graphite powder ( $\geq 99.5 \%$ pure), paraffin oil, zinc acetate dihydrate $\left(\mathrm{ZnOAc}_{2} .2 \mathrm{H}_{2} \mathrm{O}\right)(\geq 99.99 \%$ pure $)$, sucrose $\left(\mathrm{C}_{12} \mathrm{H}_{22} \mathrm{O}_{11}\right)(\geq 99 \%$ pure) and sodium hydroxide $(\mathrm{NaOH})(\geq 99 \%$ pure $)$ are procured from Merck, USA. Diethanolamine (DEA) ( $\geq 98 \%$ pure) is from Sigma-Aldrich, USA, and nitric acid $\left(\mathrm{HNO}_{3}\right)(\geq 65 \%$ pure $)$ is from S.D. Fine Chemicals, India. All chemicals are of reagent grade and 
used without additional purification. Double-distilled water from the Millipore system, Merck, USA, is used in the experiments.

\subsection{Synthesis of zinc oxide nanoparticles (ZnONPs)}

Zinc oxide nanoparticles (ZnONPs) are synthesized from zinc acetate dehydrate and sucrose using a combustion method. An aqueous solution of $10 \mathrm{~g} \mathrm{~m}^{-1}$. of zinc acetate dehydrate and $10 \mathrm{~g} \mathrm{~m}^{-1}$ of sucrose solution is prepared in $200 \mathrm{~mL}$ of Millipore water. The zinc acetate and sucrose solution in $1: 1$ volumetric ratio is homogenised by magnetic stirring. Then, $4 \mathrm{~mL}$ of DEA is added to the solution, causing precipitation. The drop-wise addition of concentrated $\mathrm{HNO}_{3}$ to the solution clears the precipitate while maintaining the $\mathrm{pH}$ of the solution at $\mathrm{pH} 2$. The resulting solution is the DEA complex of a $\mathrm{Zn}$ ion. This is completely dehydrated at $120^{\circ} \mathrm{C}$ for $30 \mathrm{~min}$ in a hot plate. After dehydration, the precursor decomposes to a black fluffy mass. The powdery mass is then calcined in an oven at $500^{\circ} \mathrm{C}$ for $4 \mathrm{~h}$. The resulting powder obtained is the carbon free zinc oxide nanocrystalline powder. According to the literature, the nanoparticles of the order of $45 \mathrm{~nm}$ are expected to form (Das et al., 2014).

\subsection{Fabrication of working electrodes for electronic tongue}

The bare CPs electrode is prepared in a regular way by mixing graphite powder $(70 \%(w / w))$ with paraffin oil $(30 \%$ $5(w / w))$ in an agata mortar for $20 \mathrm{~min}$. The paste is then packed into a glass cavity ( $1 \mathrm{~mm}$ diameter and $2 \mathrm{~mm}$ depth). The modified electrodes are prepared by mixing $0.12 \mathrm{mg}$ of $\mathrm{ZnO}$ nanoparticles and $1.12 \mathrm{mg}$ of graphite powder in $0.3 \mathrm{~mL}$ of paraffin oil in an agata mortar and mixed for $15 \mathrm{~min}$. The packing procedure is the same as that of the bare CPs electrode. Electrical contacts are established through the copper wire. The surfaces of both the electrodes are smoothed and rinsed carefully with double-distilled water prior to each measurement. When required, a new surface is obtained by pushing an excess of the paste out of the tube and polishing with a weighing paper. The fabricated electrodes are shown in Fig. 1.

\subsection{Preparation of samples}

A total of 50 samples of five different botanical origins, namely Eucalyptus (Eucalyptus globulus, 10 samples), Pumpkin (Cucurbita maxima, 10 samples), Leechi (Litchi chinensis, 10 samples), Mustard (Brassica juncea, 10 samples), and Til (Sesamum indicum, 10 samples) are analysed. All the honey samples are collected from the same geographical area, i.e. from a local apiary in Bankura district, West Bengal, India. The samples are kept at $4-5^{\circ} \mathrm{C}$ in glass vials. Prior to analyses, $20 \mathrm{~g}$ of each floral type of liquid honey sample was placed in a water bath till it liquefied, and dried.

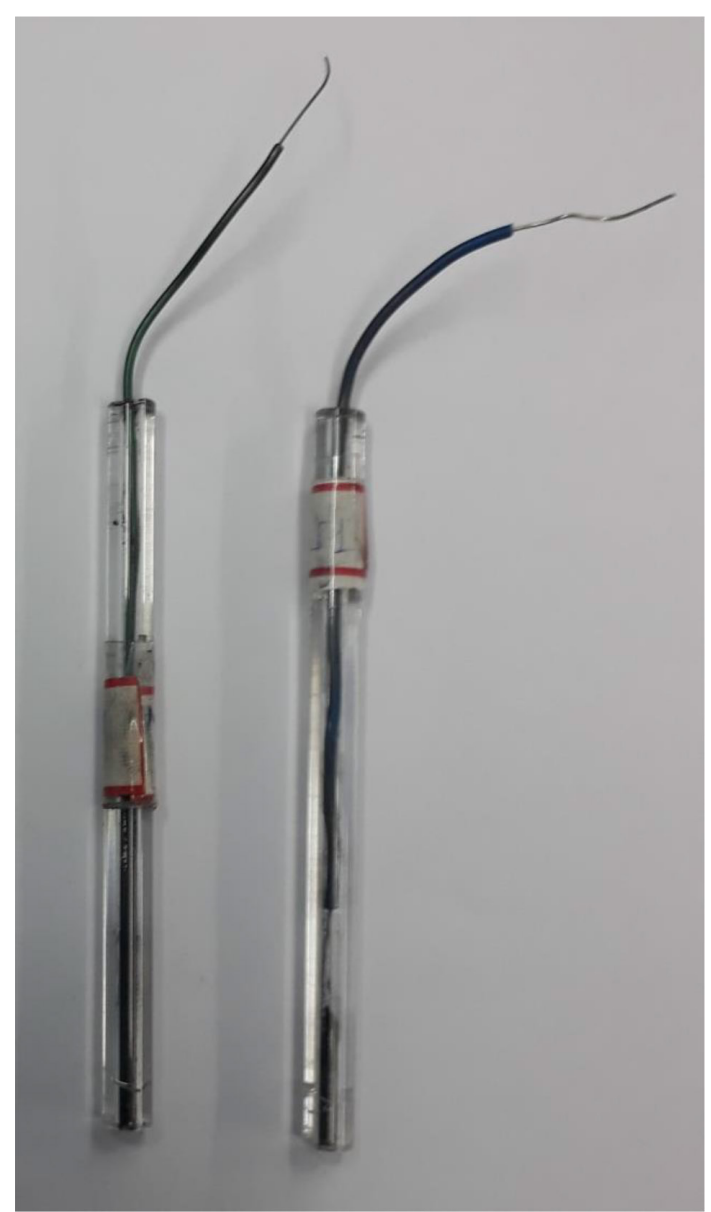

Figure 1. The fabricated electrode, CPs and ZnONPs modified CPs.

Then the same was diluted in $100 \mathrm{~mL}$ Millipore water. The solution is mechanically stirred for $0.5 \mathrm{~min}$ (Tiwari et al., 2012).

\subsection{Measurement procedure}

In each measurement cycle, $60 \mathrm{~mL}$ of each honey sample is poured in an electrochemical cell of the Gamry potentiostat. The cyclic voltammograms of each sample are recorded in the potential range of -0.45 to $0.7 \mathrm{~V}$ using a scan rate of $0.3 \mathrm{~V} \mathrm{~s}^{-1}$ for 10 cycles. Prior to each measurement, the sample solution is stirred and the three electrodes are immersed in $60 \mathrm{~mL}$ of Millipore water and rinsed. The potential of the working electrode is varied linearly with time, while the reference electrode is maintained at a constant potential. The potential is applied between the reference electrode and the working electrode and the current is measured between the working electrode and the counter electrode. The applied voltage is shown in Fig. 2a. The measured current responses are due to either oxidation or reduction of the analyte over the entire cycle at the surface of the bare CPs electrode and the modified electrode, as can be seen in Fig. $2 b$ in a monoflo- 

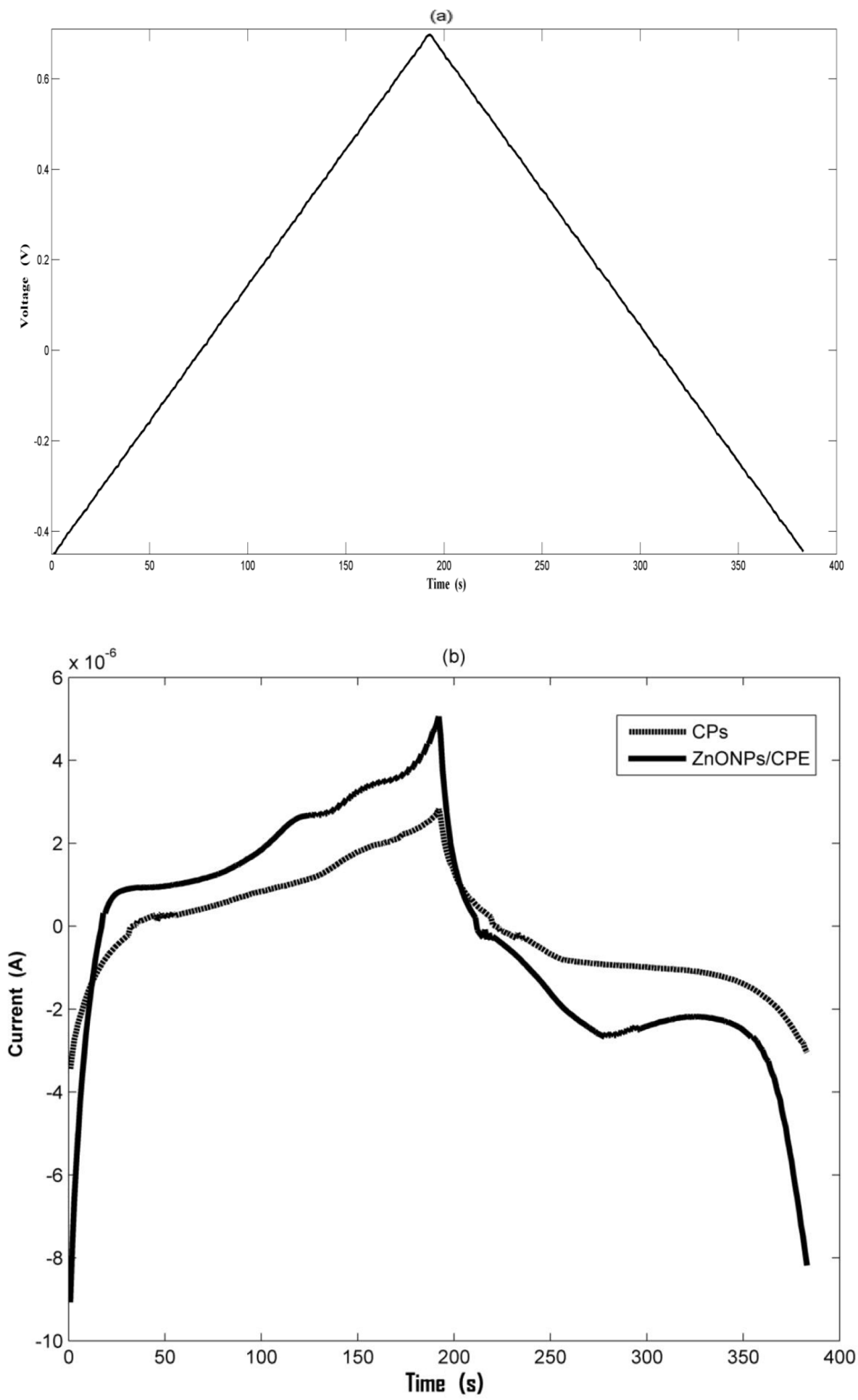

Figure 2. (a) The applied potential in the voltammogram; (b) the resultant current in the voltammogram. 


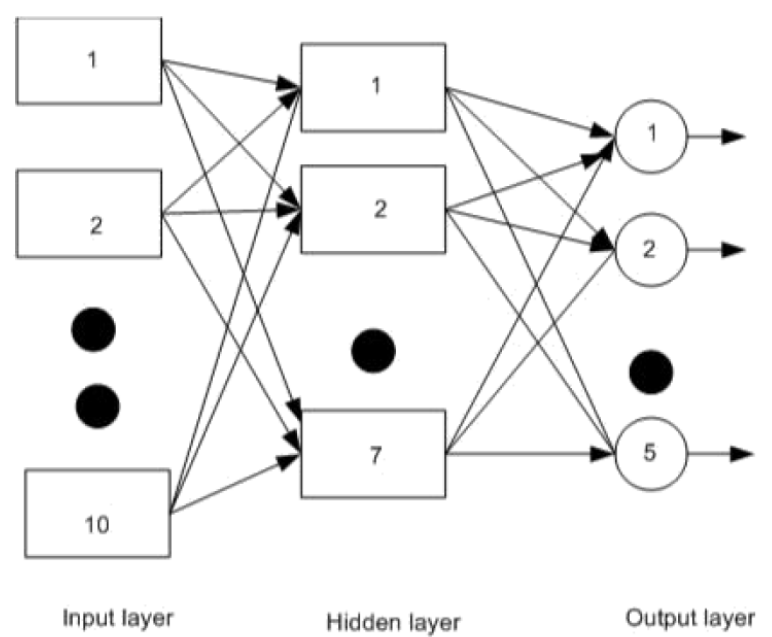

(a)

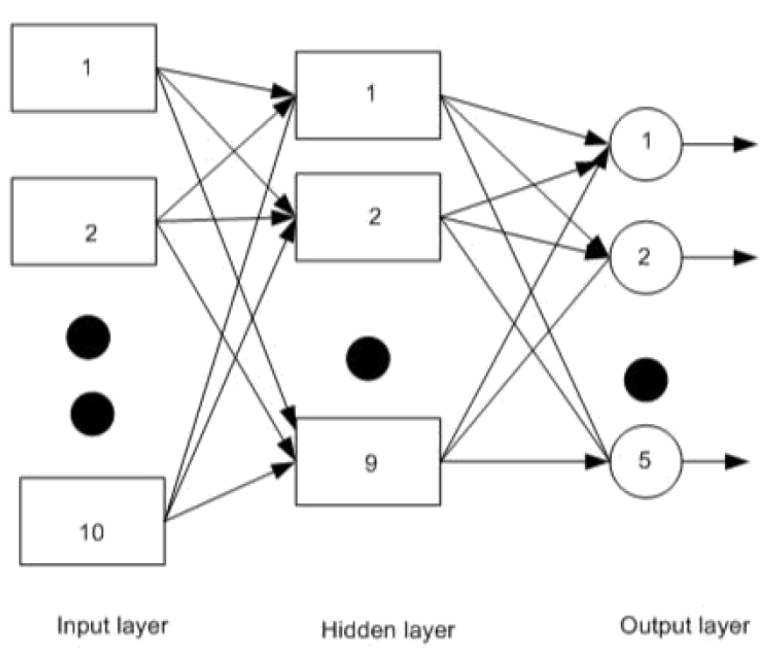

(b)

Figure 3. The pictorial representation of the (a) BPMLP and (b) RBF network models for classification of five different floral origins of honey.

ral honey sample. A large amount of data collected by both types of working electrodes is further treated using multivariate data analysis.

\subsection{Multivariate data analysis}

In order to determine the capacity of the working electrode to classify the honey samples, multivariate data analysis is used. In our experimental work we have used two working electrodes for all the 50 samples and the transient responses of the electrodes, i.e. 383 data points from a complete voltammogram of each sample and for each type of electrode are obtained. Therefore we have a data set of $50 \times 383$ for each of the working electrodes. The PCA (Pearce et al., 2003) technique is applied for qualitative analysis of the data set, whereas for quantitative analysis, an artificial neural network (ANN) (Pentos et al., 2015) is used. The data sets of each electrode have been considered for the PCA plot. The data analysis involves an initial pre-processing of the transient responses using an auto-scaling technique, i.e. each variable is mean centered and scaled to unit variance prior to PCA. In the case of back propagation multilayer perceptron (BPMLP) and radial basis function (RBF) neural network classifiers, baseline subtraction and auto-scale techniques, respectively, have been applied for pre-processing to reduce the variability of the input signal while preserving the relevant information. It reduces the training time to avoid redundancy in 10 input data and to obtain a model with better generalization ability (Tiwari et al., 2013).

PCA has been applied to verify the behaviour of the unmodified electrode and the modified electrode in the determination of the floral type of honey. The data set is generated from a large number of observations, i.e. current responses of individual floral types with each electrode. Each data set is treated with PCA to emphasize variation and pattern within a data set. The PCA plot is of vital importance as it gives a visual impression of the experimental data for each type of honey and electrode. The ANN has been used to treat the data for developing the classifier model. Multilayer perceptron has the capability to approximate arbitrary functions (Haykin, 2001). An ANN has three different layers, an input layer consisting of input signals, one or more hidden layers, and one output layer. Two different ANN frameworks have been considered, i.e. BPMLP and RBF. PCA and ANN models are implemented with Matlab version 2013a (Mathworks, Inc.) software. A pictorial representation of the BPMLP and RBF network models is shown in Fig. 3.

\subsubsection{Back propagation multilayer perceptron (BP-MLP)}

In this study a three-layer BP-MLP model with a single input layer, hidden layer and output layer has been considered. Initially the data obtained from voltammogram were treated with PCA to show the potential to discriminate the different honey samples. Then the BP-MLP was considered for classification purposes and the first 10 principal components were considered as features of the honey samples and used as the inputs of the BP-MLP. Thus the input layer consists of the first 10 principal components and the five output nodes have been considered in the output layer corresponding to five floral types of honey. During the training of the ANN model, it is observed that convergence had been found to be acceptable with one hidden layer. The number of hidden nodes in the hidden layer is taken as 7 and 900 iterations have been carried out for training. In the testing phase, after 900 iterations, the mean square error (MSE) shows over-fitting, i.e. instability. Hence network training is terminated at 900 epochs. In this study, the learning rate of the hidden layer has been 
Table 1. Artificial neural network architecture and parameters.

\begin{tabular}{lr|lr}
\hline BP-MLP & & RBF & \\
\hline Input node & 10 & Input node & 10 \\
Hidden node & 7 & Hidden node & 9 \\
$\begin{array}{l}\text { Output node } \\
\text { Learning rate } \\
\text { (hidden layer) }\end{array}$ & 5 & Output node & 5 \\
$\begin{array}{l}\text { Learning rate } \\
\text { (output layer) }\end{array}$ & 0.1 & Epoch & 900 \\
Epoch & 900 & SSE & 1.0 \\
\hline
\end{tabular}

taken at 0.2 and "TANSIG" is used as the activation function on hidden layer outputs. In the output layer, "PURELIN" is used as the activation function and the learning rate in the output layer is considered as 0.1 .

\subsubsection{Radial basis function network (RBF)}

The radial basis function is based on the theory of function approximation (Wang, 2015). Hidden nodes implement the radial basis function, i.e. Gaussian function. The output nodes implement linear summation functions as in BMLP. In the RBF neural network model, the number of input nodes is 10 and the output layer has 5 nodes. The best output has been obtained with a single hidden layer with nine nodes. The parameters chosen for two ANN networks are shown in Table 1. The steps of data analysis are shown in Fig. 4.

\section{Results and discussions}

The objective of the work was to evaluate the capability of the ZnONPs modified CPs electrode of the voltammetric electronic tongue system in discriminating different floral varieties of honey. In this direction, we focused our attention on the newly developed surface morphology which is responsible for the electrocatalytic behaviour and its capability in the electronic tongue response for the $\mathrm{ZnONPs}$ modified CPs electrode sensor in honey floral discrimination.

\subsection{Optimization of amount of ZnONPs in the CPs electrode}

The number of metal oxide modifiers influences the performance of the working electrode in an electronic tongue. It is observed that the number of zinc oxide nanoparticles in the CPs electrode influences the electo-oxidation of honey as examined by $\mathrm{CV}$ studies. On increasing the amount of ZnONPs in CPs, the sensitivity of the sensor is increased. A ratio of $1: 9$ is found to be most suitable and, above this ratio, no further increase in redox peaks is observed.

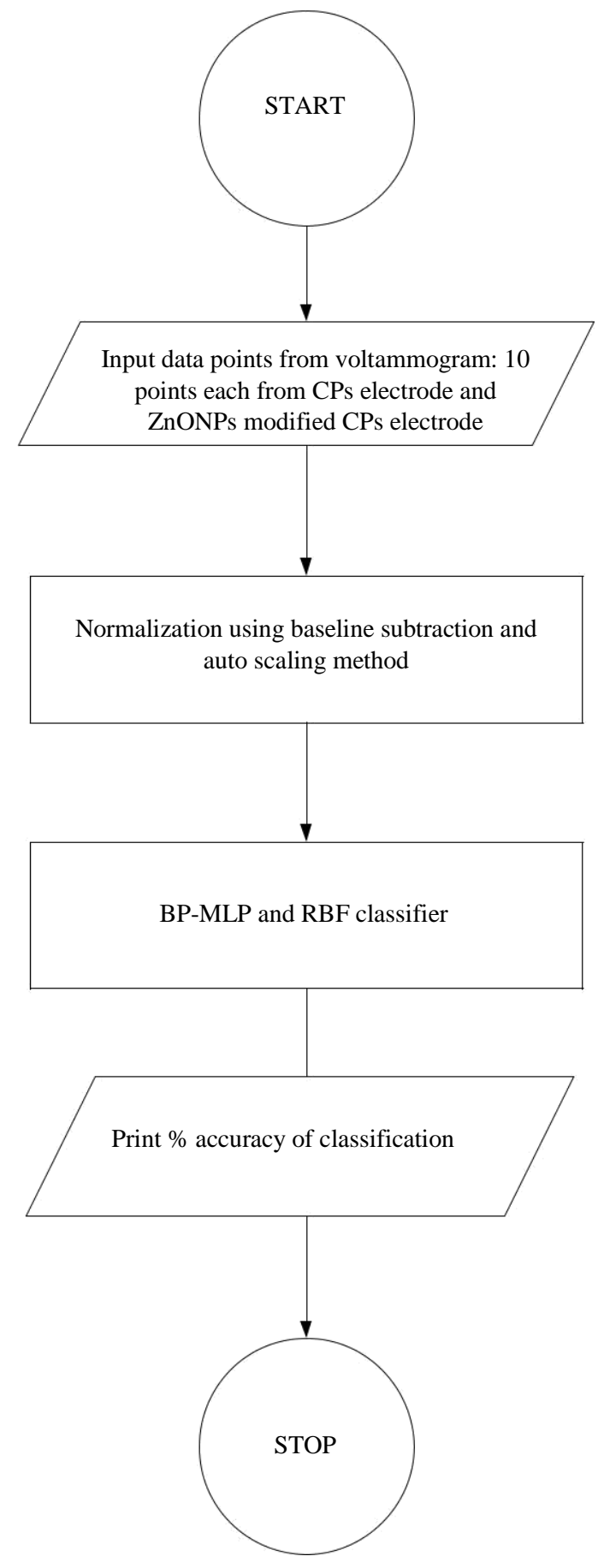

Figure 4. Flowchart for data analysis using neural networks, i.e. the input layer consists of the first 10 principal components and the five output nodes correspond to five floral types of honey.

\subsection{CV of bare CPs and the modified electrode}

The electrochemical behaviours of the carbon paste electrode (CPs electrode) and zinc oxide CPs electrode (ZnONPs modified CPs) have been studied using cyclic voltammetry. Fig- 

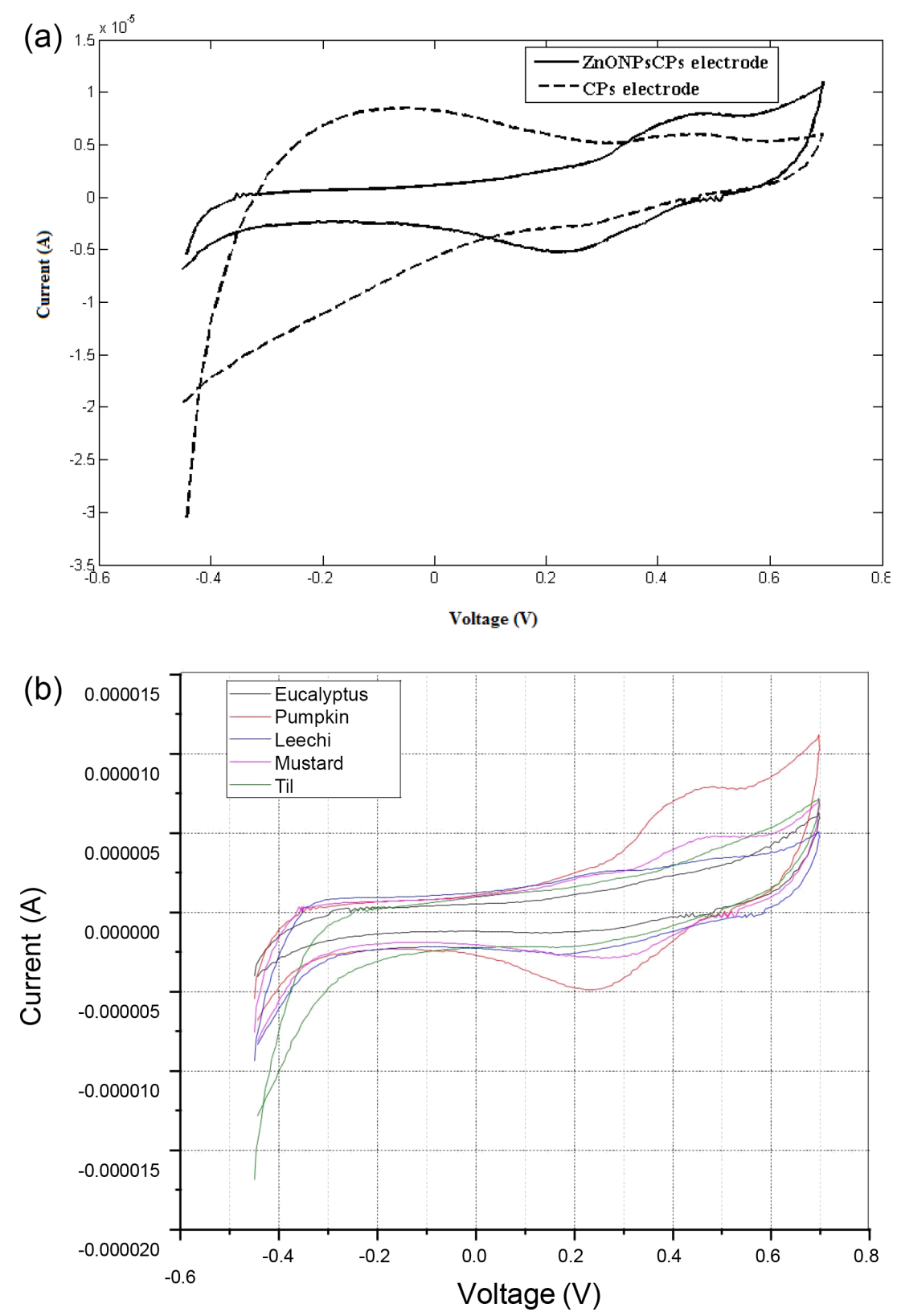

Figure 5. (a) Cyclic voltammograms of the Cps electrode and ZnONPs modified Cps electrode in the Eucalyptus floral type of honey at $0.3 \mathrm{~V} \mathrm{~s}^{-1}$ in a potential window from the -0.45 to $0.7 \mathrm{vs}$. $\mathrm{Ag} / \mathrm{AgCl}$ reference electrode. (b) Cyclic voltammograms of the ZnONPs modified CPs electrode for five different floral types of honey.

ure 5a shows the voltammograms of the bare CPs electrode and ZnONPs modified CPs electrode for the honey sample. The electrochemical oxidation of all the floral types of honey is observed using the scan rate of $0.3 \mathrm{~V} \mathrm{~s}^{-1}$ over a potential range of -0.45 to $0.7 \mathrm{~V}$. In Fig. 5a, CVs of bare CPs indicate no prominent redox peak for different floral types of honey as compared to the CV response of the honey samples using modified electrodes, which shows prominent peaks. The redox peaks are not attributed to any particular analyte of honey, but to the overall complex activity of a honey sample with that of the electrode surface. In the case of the ZnONPs modified CPs electrode, the background current is apparently larger and indicates that the effective electrode surface area is significantly enhanced due to modification. This result proves that the electro-catalytic effect of the electrode surface and the electrochemical sensor signal with each floral type of honey can be improved when metal oxide modification is applied to a CPs electrode. Table 2 shows a cathodic and anodic peak current observed for the ZnONPs modified CPs electrode of each floral type of honey. In Fig. 5b, the 
(a)

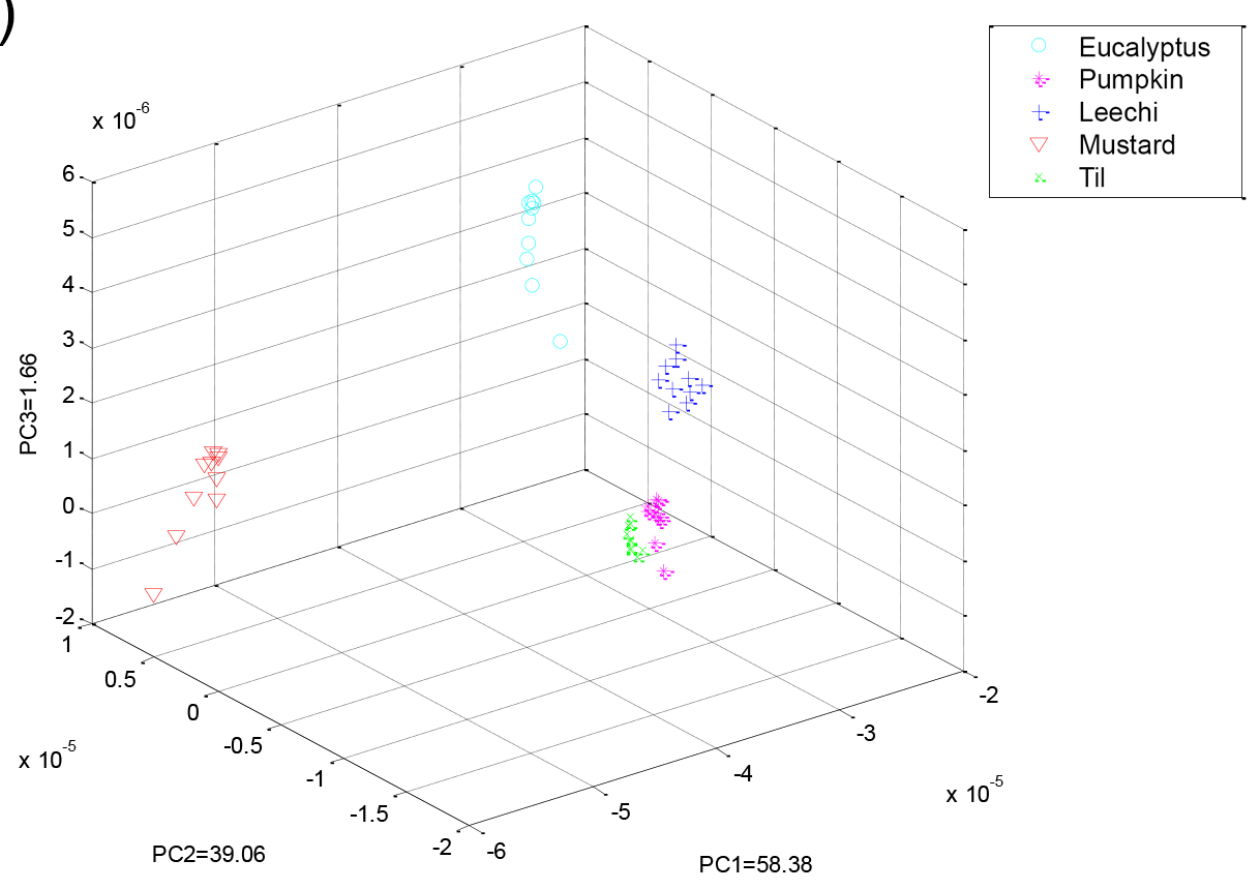

(b)

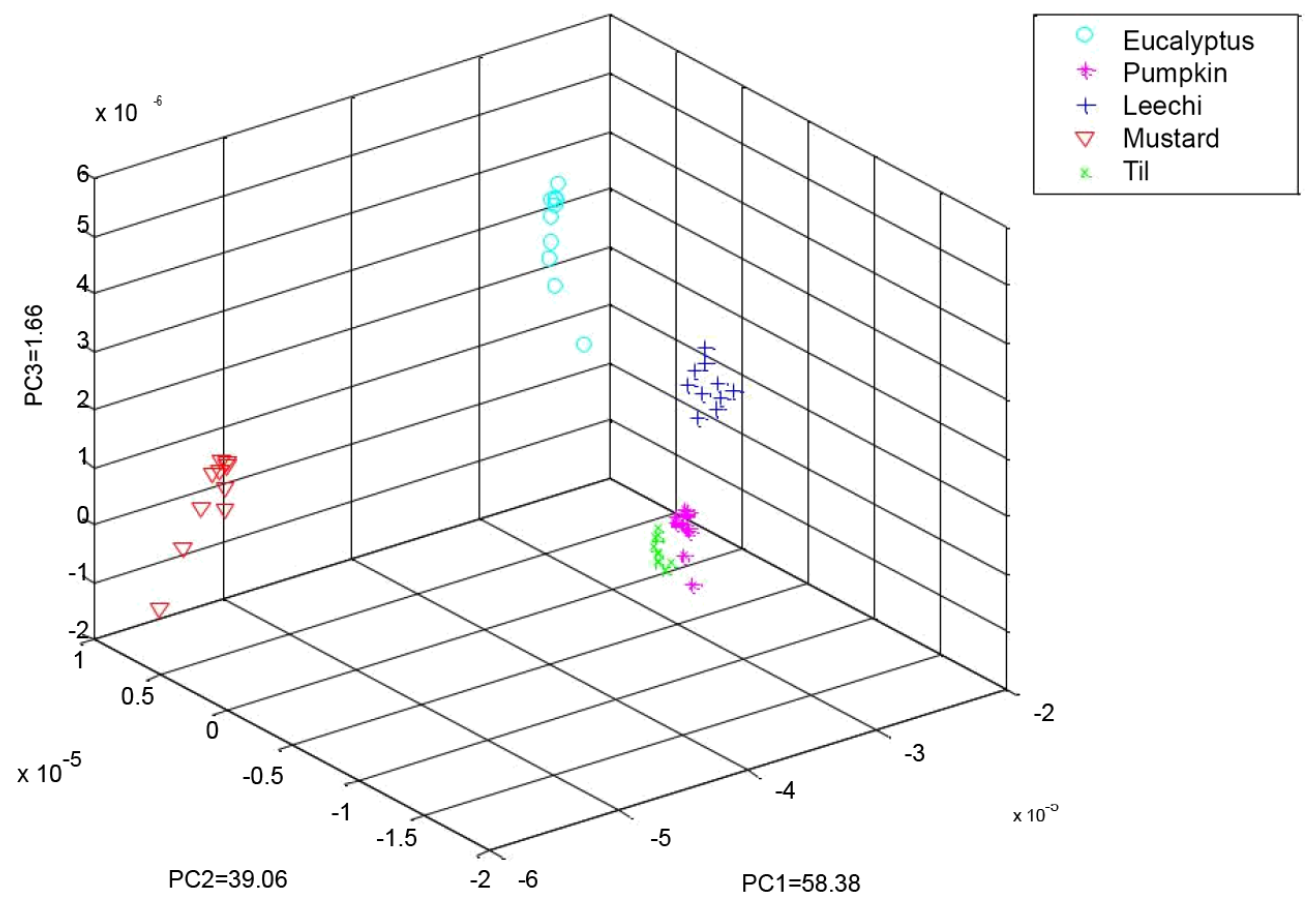

Figure 6. (a) PCA plots of honey samples using a bare CPs electrode; (b) $\mathrm{ZnO}$ nanoparticle modified CPs electrode. 
Table 2. Cathodic and anodic peak current observed for the ZnONPs modified CPs electrode of each floral type of honey.

\begin{tabular}{lrr}
\hline Sample & Ipa(A) & Ipc(A) \\
\hline Eucalyptus & 2.19 & -1.13 \\
Pumpkin & 7.83 & -4.99 \\
Leechi & 2.58 & -2.63 \\
Mustard & 4.74 & -2.9 \\
Til & 2.14 & -2.24 \\
\hline
\end{tabular}

ZnONPs modified Cps electrode exhibits separate cathodic and anodic peaks for each floral origin of honey. As honey has a complex composition, the electrode does not act particularly to any single compound. Thus, each data point on the voltammograms contains important information on the sample as a whole.

\subsection{Reproducibility and stability}

The reproducibility of the modified sensors is investigated by analysing each floral type for five times with each electrode. To ascertain the reproducibility results, the cyclic voltammetric experiments are carried out using the modified electrodes under similar conditions. The peak currents for each floral type have not changed much even after 90 days. A small decrease in current sensitivity is observed with a relative standard deviation (RSD) of about 2 and $2.5 \%$, respectively, for the two types of electrodes fabricated. This indicates the good sensor-to-sensor reproducibility. The stability is examined for each sensor for 10 times in the same concentration of the honey sample. A small change in the CV transient signal is observed after a few months. This shows the long-term stability of the modified electrode.

\subsection{Data exploration using PCA for qualitative analysis}

The non-supervised PCA method is applied to CPs and ZnONPs modified CPs electrodes and signal profiles are measured in different honey samples to display its variability. The PCA is carried out with 50 samples of five different floral types, that is, 10 samples of each type. The analysis is independent of the sample class information and facilitates visualization of clusters of samples and their floral origin. The score plots with a CPs sensor and a ZnONPs modified CPs electrode are presented in Fig. 6a and b, respectively. The PCA plot of the CPs electrode shows $98.75 \%$ of total variance as compared to $99.10 \%$ of the ZnONPs modified CPs electrode. As can be inferred from the plots in Fig. 6ab, 50 samples of honey could be separated into five different groups, confirming the performance of the individual sensors used in the electronic tongue system. The PCA plot of the ZnONPs modified CPs electrode shows partial overlapping between pumpkin and Leechi floral type of honey samples with $98.76 \%$ variances. The cumulative variances of a metal
Table 3. Classification results using the BP-MLP and RBF network model.

\begin{tabular}{lrr}
\hline Floral source of honey & \multicolumn{2}{c}{ Correct classification \% } \\
\cline { 2 - 3 } & BP-MLP & RBF \\
\hline Eucalyptus & 96.25 & 95.75 \\
Leechi & 94.52 & 90.50 \\
Pumpkin & 92 & 89.45 \\
Mustard & 94 & 90 \\
Til & 95 & 86 \\
\hline
\end{tabular}

oxide based Cps electrode are higher as compared to the bare CPs electrode and metal electrode (Pearce et al., 2003). Also, the results show a class separability index (Tiwari et al., 2013) value of 6.37 for the CPs electrode as compared to the nano-metal oxide based CPs, which has a value of 8.09, in the case of ZnONPs, respectively. This improvement shows the utility of a metal oxide based modified Cps electrode. This fact suggests that the metal oxide modified Cps working electrodes in a voltammetric based electronic tongue can be used for more efficient floral identification with better clustering as compared to the bare Cps electrode.

\subsection{ANN models for quantitative analysis}

The classification of five different floral types of honey has been done using two architectures of ANN, i.e. BP-MLP and RBF. For each of the 50 samples of honey, the first 10 principal components (PCs) have been considered as input variables. Thus, the obtained total data set is of size $50 \times 10$. The data set is divided into a training set of size $30 \times 10(60 \%)$ and a test set of size $20 \times 10(40 \%)$. The performances of the two ANN models for classifying five different floral types are shown in Table 3.

\section{Conclusions}

In this study, a simple fabrication method of a ZnONPs modified CPs electrode is demonstrated for the discrimination of honey from different floral origins. The modified electrode is prepared by using a facile combustion route in order to catalyse the electro-active compounds present in honey. From cyclic voltammetry, it is confirmed that higher anodic current densities can be achieved on the surface of the ZnONPs electrode than at the unmodified CPs electrode. The classification performance by BP-MLP and RBF models for building the electronic tongue response model shows an average of 94.34 and $90.34 \%$ accuracy, respectively. However, in order to use voltammetric sensors in the electronic tongue as a routine methodology, it is recommended to improve the sensor system by including different metal oxide NPs in the CPs electrodes for better discrimination of compositional variation. Compared with platinum and bare CPs sensors, the $\mathrm{ZnO}$ 
modified CPs electrode demonstrates better differentiation amongst the five floral types of honey (Eucalyptus, Pumpkin, Leechi, Mustard and Til). This sensor also exhibits good stability and reproducibility.

Data availability. A data sheet containing electronic tongue responses obtained from a $\mathrm{ZnO}$ NPs CPs electrode for different floral origin of honey is available as a Supplement to this paper.

Supplement. The supplement related to this article is available online at: https://doi.org/10.5194/jsss-7-319-2018-supplement.

Competing interests. The authors declare that they have no conflict of interest.

Acknowledgements. The authors would like to express their thanks to the "Women Madhu Society", Bankura, West Bengal, India, for providing the honey samples.

Edited by: Anita Lloyd Spetz

Reviewed by: three anonymous referees

\section{References}

Afghani, A., Gomar, F., and Madrakian, T.: $\mathrm{CoFe}_{2} \mathrm{O}_{4}$ nanoparticles modified carbon paste electrode for simultaneous detection of oxycodone and codeine in human plasma and urine, Sensor Actuat. B-Chem., 233, 263-271, https://doi.org/10.1016/j.snb.2016.04.067, 2016.

Anklam, E.: A review of the analytical methods to determine the geographical and botanical origin of honey, Food Chem., 63, 549562, https://doi.org/10.1016/S0308-8146(98)00057-0, 1998.

Apetrei, I. M. and Apetrei, C.: Application of voltammetric e-tongue for the detection of ammonia and putrescine in beef products, Sensor Actuat. B-Chem., 234, 371-379, https://doi.org/10.1016/j.snb.2016.05.005, 2016.

Ares, M., Soto, M. E., Nozal, M. J., and Bernal, J. L.: Determinations of Resveratrol and Piceid Isomers in bee pollen by liquid chromatography coupled to electrospray ionizationmass spectrometry, Food Anal. Methods, 8, 1565-1575, https://doi.org/10.1007/s12161-014-0048-8, 2015.

Ball, D. W.: The chemical composition of honey, J. Chem. Educ., 84, 1643, https://doi.org/10.1021/ed084p1643, 2007.

Belay, A., Haki, G. D., Birringer, M., Borck, H., Addi, A., Baye, K., and Melaku, S.: Rheology and botanical origin of Ethiopian monofloral honey, Food Sci. Tech., 75, 393-401, 2017.

Bijad, M., Karimi-Maleh, H., and Khalilzadeh, Md. A.: Application of ZnO/CNTs Nano composite Ionic Liquid Paste electrodes as a sensitive voltammetric sensor for determination of Ascorbic acid in food samples, Food Anal. Meth., 6, 1639-1647, https://doi.org/10.1007/s12161-013-9585-9, 2013.

Bueno-Costa, F. M., Zambiazi, R. C., Bohmer, B. W., Chaves, F. C., Silva, W. P., Zanusso, J. T., and Dutra, I.: Antibacterial and antioxidant activity of honey from the state of Rio
Grande do Sul, Brazil, LWT-Food Sci. Technol., 65, 333-340, https://doi.org/10.1016/j.lwt.2015.08.018, 2015.

Codex Alimentarius Commission: Draft revised standard for standard for honey (at step 10 Of the Codex procedure), in: Report Of The Seventh Session Of The Codex Committee On Sugars, Alinorm 01/25, 19-26, 2001.

Cavazza, A., Corradini, C., Musci, M., and Salvadeo, P.: Highperformance liquid chromatographic phenolic compound fingerprint for authenticity assessment of honey, J. Sci. Food Agr., 93, 1169-1175, 2013.

Ceto, X., Gonzale-Calabuig, A., Crespo, N., Perez, S., Capdevila, J., Puig-Pujol, A., and Del-Vall, M.: Electronic tongues to assess wine sensory descriptors, J. Talanta, 62, 218-224, 2017.

Chua, L. S., Abdul-Rahaman, N. L., Sarmidi, Md. R., and Aziz, R.: Multi-elemental composition and physical properties of honey samples from Malaysia, Food Chem., 135, 880-887, 2012.

Das, S. C., Sadani, K., Bandyopadhyay, R., and Pramanik, P.: Characteristics of Molybdenum doped Zinc Oxide Nanoparticles Chemoresistor Pellets Towards Black Tea-Biochemicals, Int. J. Adv. Eng. Sci. Tech., 120-131, ISSN: 2319-1120/V3N3:120131, 2014.

Dias, L. G., Veloso, A. C. A., Sousa Mara, E. B. C., Estevinho, L., Machado Adelio, A. S. C., and Peres Antonio, M.: A novel approach honey pollen profile assessment using an electronic tongue and chemometric tools, Anal. Chim. Acta, 900, 36-45, 2015.

Dinca, O. R., Elena, R., Popescu, R., Costinel, D., and Radu, G. L.: Geographical and botanical origin discrimination of Romanian Honey using complex stable isotope data and Chemmometrics, Food Anal. Meth., 8, 401-412, 2015.

El Sohaimy, S. A., Masry, S. H. D., and Shehata, M. G.: Physicochemical characteristics of honey from different origins, Ann. Agr. Sci., 60, 279-287, 2015.

Feas, X., Pires, J., Iglesias, A., and Estevinho, M. L.: Characterization of artisanal honey produced on the Northwest of Portugal by melissopalynological and physic-chemical data, Food Chem. Toxicol., 48, 3462-3470, 2010.

Fernandez Garcia, M. and Rodriguez, J. A.: Metal oxide nanoparticles, in: Nanomaterial: Inorganic and Bioinorganic Perspectives, Brookhave National Laboratory, No. BNL-79479-2007BC, 2007.

Haykin, S.: Neural Networks - A comprehensive foundation, 2nd edn., Pearson Education, Asia, 2001.

Jiang, T.-F., Chong, L., Yue, M.-E., Wang, Y.-H., and Lv, Z.-H.: Separation and determination of carbohydrate in food samples by capillary electrophoresis using dynamically coating the capillary with indirect UV detection, Food Anal. Method., 8, 2588-2594, https://doi.org/10.1007/s12161-015-0157-z, 2015.

Kadri, S. M., Zaluski, R., and Orsi, R. O.: Nutritional and mineral contents of honey extracted by centrifugation and pressed processes, Food Chem., 218, 237-241, 2017.

Karabagias, I. K., Badeka, A. V., Kontakos, S., Karabournioti, S., and Kontominas, M. G.: Botanical discrimination of Greek unifloral honeys with physico-chemical and chemometric analyses, Food Chem., 165, 181-190, 2014a.

Karabagias, I. K., Vavoura, M. G., Nikolaou, C., Badeka, A. V., Kontakos, S., and Kontominas, M. G.: Floral authentication of Greek unifloral honeys based on the combination of pheno- 
lic compounds, physicochemical parameters and chemometrics, Food Res. Int., 62, 753-760, 2014b.

Karim-Nezhad, G., Khorablou, Z., Zamani, M., Sayed Dorraji, P., and Alamgholiloo, M.: Voltammetric sensor for tartrazine determination in soft drinks using poly (p-amino benzene sulfonic acid) /zinc oxide nanoparticles in carbon Paste electrode, J. Food Drug Anal., 25, 293-301, https://doi.org/10.1016/j.jfda.2016.10.002, 2016.

Kaskoniene, V., Ruockuviene, G., and Kaskonas, P.: Chemometric analysis of bee pollen based on volatile and phenolic compound composition and antioxidant properties, Food Anal. Meth., 8, 1150-1163, 2015.

Legin, A., Rudnitskaya, A., and Vlasov, Y.: Electronic tongues: new analytical perspective for chemical sensors, Comprehensive Analytical Chemistry, XXXIX, 437-486, ISSN:0166-526X, 2003.

Mandal, M. D. and Mandal, S.: Honey: its medicinal property and antibacterial activity, Asian Pac. J. Trop. Biomed., 1154-160, 2011.

Missio da Silva, P., Gauche, C., Gonzaga, L. V., Carolina, A., Costa, O., and Fett, R.: Honey chemical composition, stability and authenticity, Food Chem., 196, 309-323, 2016.

Oryan, A., Alem Zadeh, E., and Moshin, A.: Biological properties and therapeutic activities of honey in wond healing: A narrative review and meta-analysis, J. Tissue Viability, 25, 98-118, 2016.

Ozcan, M. M. and Olmez, C.: Some qualitative properties of different monofloral Honeys, Food Chem., 163, 212-218, 2014.

Pearce, T. C., Schiffman, S. S., Nagle, H. T., and Gardner, J. W.: Handbook of Machine Olfaction, Wiley-VCH Verlag GmbH \& Co. KGaA, 2003.

Pentos, K., Luczycka, D., and Kaplon, T.: The identification of relationship between selected honey parameters by extracting the contribution of independent variables in a neural network model, Eur. Food Res. Technol., 241, 793-801, 2015.

Peris, M. and Gilabert, L. E.: Electronic noses and tongues to assess food authenticity and adulteration, Trends Food Sci. Tech., 58, 40-54, 2016.

Pohl, P., Stecka, H., Sergiel, I., and Jamroz, P.: Different aspects of elemental analysis of honey by flame atomic absorption and emission spectrometry: A review, Food Anal. Meth., 5, 737-751, https://doi.org/10.1007/s12161-011-9309-y, 2011.

Rezic, I., Horvat, A. J. M., Babi, C. S., and Kastelan-Macan, M.: Determination of pesticides in honey by ultrasonic solvent extraction and thin-layer chromatography, Ultrason. Sonochem., 12, 477-481, 2005.

Rodriguez Reinoso, F.: The role of carbon materials in heterogeneous catalysis, Carbon, 36, 159-175, 1998.

Siddiqui, A. J., Musharraf, S. G., Choudhary, M. I., and Rahman, A.: Application of analytical methods in authentication and adulteration of honey, Food Chem., 217, 687-698, 2017.

Skrzypczynska, K., Kusmierek, K., and Swiatkowski, A.: Carbon paste electrodes modified with various carbonaceous materials for the determination of 2,3-dichlorophenoxyacetic acid by differential pulse voltammetry, J. Electroanal. Chem., 766, 8-15, 2016.

Soto, V. C., Maldonado, I. B., Jofre, V. P., Galmarini, C. R., and Silva, M. F.: Direct analysis of nectar and floral volatile organic compounds in hybrid onions by HS- SPME/GC-MS: Relationship with pollination and seed production, Microchemical J., 122, 110-118, 2015.
Sousa, M. E., Dias, L. G., Veloso, A. C., Estevinho, L., Peres, A. M., and Machado, A. A.: Practical procedure for discriminating monofloral honey with a broad pollen profile variability using an electronic tongue, J. Talanta, 128, 284-292, 2014.

Svancara, I. and Schachl, K.: Testing of unmodified carbon paste electrodes, Chemicke Listy, 93, 490-499, 1999.

Svancara, I., Vytras, K., Barek, J., and Zima, J.: Carbon Paste electrodes in Modern Electroanalysis, CRC Cr. Rev. Anal. Chem., 31, 311-345, 2001.

Svancara, I., Vytras, K., Kalcher, K., Walcarius, A., and Wang, J.: Carbon paste electrodes in Facts, Numbers, and Note: A review on the occasion of the 50-years Jubilee of carbon paste in electrochemistry and electroanalysis, Electroanalysis, 21, 7-28, 2008.

Tavakolian, E., Tashkhourian, J., Razmi, Z., Kazemi, H., and Hosseini Sarvari, M.: Ethanol electro-oxidation at carbon paste electrode modified with Pd-ZnO Nanoparticles, Sensor Actuat. BChem., 230, 87-93, 2016.

Terbouche, A., Lameche, S., Ramdane-Terbouche, C. A., Guerniche, D., Lerari, D., Lerari, D., and Hauchard, D.: A new electrochemical sensor based on carbon paste Electrode/Ru (III) complex for determination of nitrite: Electrochemical impedance and cyclic voltammetry measurements, Measurement, 92, 524533, 2016.

Tiwari, K., Tudu, B., Bandyopadhyay, R., and Chatterjee, A.: Discrimination of monofloral honey using cyclic voltammetry, Proceedings IEEE-NCETACS, 132-136, 2012.

Tiwari, K., Tudu, B., Bandyopadhyay, R., and Chatterjee, A.: Identification of monofloral honey using voltammetric electronic tongue, J. Food Engineering, 117, 206-210, 2013.

Ulloa, P. A., Guerra, R., Cavaco, A. M., Figueira, A. M., and Brigas, A. F.: Determination of the botanical origin of honey by sensor fusion of impedance e-tongue and optical spectroscopy, Comput. Electron. Agr., 94, 1-11, 2013.

Wang, P.: Bio inspired smell and taste sensors, Springer, ISBN: 7030457617, 2015.

Wei, Z. and Wang, J.: Classification of monofloral honeys by voltammetric electronic tongue with chemometrics method, Electrochim. Acta, 56, 4907-4915, 2011.

Wei, Z. and Wang, J.: The evaluation of sugar content and firmness of non- climatic pears based on voltammetric electronic tongue, J. Food Eng., 117, 158-164, 2013.

Wei, Z. and Wang, J.: Tracing floral and geographical origins of honeys by potentiometric and voltammetric electronic tongue, Comput. Electron. Agr., 108, 112-122, 2014.

Woodcock, T., Downey, G., Kelly, J. D., and Donnell, C.: Geographical classification of honey samples by near-infrared spectroscopy: a feasibility study, J. Agri. Food Chem., 55, 9128 9134, 2007.

Yousuf, F. A., Mehmood, M. H., Malik, A., Siddiqui, R., and Khan, N. A.: Antiacanthamoebic properties of natural and marketed honey in Pakistan, Asian Pac. J. Trop. Biomed., 6, 967-972, 2016.

Zheng, X., Zhao, Y., Wu, H., and Feng, J.: Origin identification and quantitative analysis of honey by nuclear magnetic resonance and chemometrics techniques, Food Anal. Meth., 9, 1470-1479, https://doi.org/10.1007/s12161-015-0325-1, 2015. 